\title{
Structural basis for DAXX interaction with ATRX
}

\section{Dear Editor,}

Alpha-thalassemia/mental retardation syndrome $X$-linked protein (ATRX) is a member of the switch 2/sucrose nonfermentable 2 (SWI2/SNF2) family of chromatin-remodeling proteins (Clynes et al., 2013; Dyer et al., 2017). ATRX deposits histone variant $\mathrm{H} 3.3$ into heterochromatin loci with the cooperation of an H3.3-specific chaperone, the deathdomain associated protein (DAXX) (Goldberg et al., 2010; Law et al., 2010; Lewis et al., 2010). Loss of ATRX or DAXX leads to an increased DNA damage response, activation of the alternative lengthening of telomeres (ALT) pathway, and genomic instability (Dyer et al., 2017). Consequently, genome sequencings have identified ATRX and DAXX mutations in a variety of cancers (Watson et al., 2015). Due to the important roles of the DAXX-ATRX complex in the maintenance of heterochromatin structure and stability, the structural studies of ATRX and DAXX have been extensively carried out.

ATRX contains two structural domains. One is the N-terminal ADD (ATRX-DNMT3-DNMT3L) domain that specifically recognizes $\mathrm{H} 3$ lysine 9 trimethylation (H3K9me3) (Iwase et al., 2011). The other one is C-terminal ATP-dependent chromatin-remodeling domain, which has not been structurally characterized. DAXX also contains two structural regions. One is N-terminal DAXX helical bundle (DHB) domain, which has been shown to interact with RASSF1C (Ras-association domain family 1 isoform C), P53 and MDM2 (mouse double minutes 2 homolog) (Escobar-Cabrera et al., 2010). The other one is histone binding domain (HBD), responsible for specific recognition of H3.3-H4 (Elsasser et al., 2012; Liu et al., 2012). However, the manner in which DAXX interacts with ATRX to orchestrate the histone chaperone activity of DAXX and the chromatin remodeling activity of ATRX remains largely unclear.

In the present study, we first dissected the interaction between DAXX and ATRX. The DAXX helical bundle (DHB) domain has been shown to interact with two modules of ATRX (Tang et al., 2004). Residues between 1,189 and 1,326 of ATRX serve as the dominant binding module for DAXX, and residues 321-865 of ATRX may constitute a secondary DAXX-interacting module (Tang et al., 2004). Here we used isothermal titration calorimetry (ITC) to evaluate the contribution of each ATRX module to the DAXXATRX interaction. We found that ATRX ${ }_{1,188-1,326}$ interacts with DAXX undergoes no detectable binding to DAXX $X_{D H B}$ (Fig. S1A). Therefore, we focused on ATRX ${ }_{1,188-1,326}$ for further investigation. We generated a panel of ATRX fragments spanning 1,188-1,326 and examined their binding capacities with DAXX $X_{\text {DHB }}$ (Fig. S1B). A minimal ATRX fragment consisting of residues 1,260-1,289 was both necessary and sufficient to interact with DAXX $\mathrm{DHB}_{\mathrm{DHB}}$ (Fig. S1B and S1C). Hereafter, we will refer to ATRX ${ }_{1,260-1,289}$ as the DAXX-binding motif of ATRX (ATRX

We determined the structure of the DAXX $X_{D H B}-A T R X_{D B M}$ complex at a resolution of $2.2 \AA$ using single-wavelength anomalous dispersion with selenomethionine-substituted crystals (Table S1). The structure has been refined to an $R$-value of $18.7 \%\left(R_{\text {free }}=21.9 \%\right)$ with good geometry. The electron density map allowed us to trace most of the complex without much ambiguity (Fig. S2A). The final refined model covered DAXX residues 57-141 and ATRX residues 1,267-1,284. DAXX $X_{\mathrm{DHB}}$ forms an elongated helix bundle with four antiparallel packed helices $\alpha 1, \alpha 2, \alpha 4$, and $\alpha 5$ (Fig. 1B). $\alpha 3$ is a short helix connecting $\alpha 2$ and $\alpha 4$, and it crosses the base of the helical bundle. ATR $X_{\mathrm{DBM}}$ exists as a long amphipathic helix (residues 1,269-1,283) lying along the cleft between helices $\alpha 2$ and $\alpha 5$ of DAXX $A_{T R X_{D B M}}$ binding does not induce large conformational change in $D A X X_{D H B}$, because the DAXX $X_{D H B}$ structure in the complex is almost identical to the previously determined

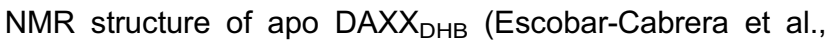
2010), with a root-main-square deviation (rmsd) value of $1.0 \AA$ for 83 equivalent $C \alpha$ pairs.

The interaction between $\mathrm{DAXX} \mathrm{XHB}_{\mathrm{DH}}$ and ATRX $\mathrm{XBBM}_{\mathrm{D}}$ is predominantly mediated by hydrophobic contacts. Four nonpolar residues (A1272, L1276, L1277, and I1280) in the center of the ATRX $X_{D B M}$ helix constitute a hydrophobic core that fits snugly into a shallow groove in DAXX $\mathrm{DHB}_{\mathrm{DH}}(\mathrm{Fig} .2 \mathrm{~A})$. The side chains of these residues make close contacts with a panel of hydrophobic amino acids, including V84, F87, Y124, V125, and I127 of DAXX (Fig. 2A). Consistent with the structural model, mutations of any of the hydrophobic residues on ATRX destabilized the DAXX ${ }_{D H B}-A T R X_{D B M}$ interaction (Fig. 2B). In particular, mutations in ATRX ${ }^{L 1276}$ and 
ATRX ${ }^{L 1280}$ had the most disruptive effects, and double mutants (ATRX ${ }^{\mathrm{L} 1276 R / L 1280 R}$ and ATRX ${ }^{\mathrm{L} 1276 \mathrm{~L} / \mathrm{L} 1280 \mathrm{Q}}$ ) completely abolished the interaction with $D A X X_{D H B}$ (Fig. 2B). Similarly, the hydrophobic residues on $\mathrm{DAX} \mathrm{X}_{\mathrm{DHB}}$ are also crucial to the binding of ATRX (Fig. 2C). Point mutations of these hydrophobic residues impaired the $\mathrm{DAX} \mathrm{DHB}_{\mathrm{DH}^{-}}$ ATRX $_{\text {DBM }}$ interactions, and a DAXX double mutant (F87A/ Y124A) completely lost its ability to bind to ATRX (Fig. 2C). These data indicate that the hydrophobic interactions are the major driving force for the binding of $A T R X_{D B M}$ to $D A X X_{D H B}$. These hydrophobic residues in DAXX and ATRX are well conserved across many species (Fig. S2B and S2C), suggesting that DAXX-ATRX in other species may also adopt the same interaction mode. The only exception is the Drosophila counterparts of DAXX and ATRX, dDAXX and dXNP. For example, DAXX F87 is replaced with a glutamate in dDAXX, and ATRX 11280 by an arginine in dXNP (Fig. S2B and $S 2 C$ ). These differences may severely impair the interaction between dXNP and dDAXX. Whether and how the Drosophila counterparts interact with each other remains to be determined.
Complementary with the hydrophobic contacts, a series of salt bridges and hydrogen-bonding interactions further strengthened the interactions between $D_{D A X} X_{D H B}$ and $A T R X_{D B M}$. The carboxylate group of ATRX ${ }^{E 1268}$ engages in a salt-bridge interaction with $\mathrm{DAX} \mathrm{X}^{\mathrm{K} 122}$, while $A T R \mathrm{X}^{\mathrm{E} 1279}$ coordinates a salt bridge with DAXX ${ }^{\mathrm{R} 91}$ (Fig. 2D). In addition, the carboxamide group of ATRX ${ }^{\mathrm{N} 1269}$ forms two hydrogen bonds with the backbone carbonyl of DAXX ${ }^{A 121}$ and the backbone amide of $\mathrm{DAXX} \mathrm{Y}^{\mathrm{124}}$ (Fig. 2D). The carbonyl of $A T R X^{A 1272}$ forms a hydrogen bond to DAXX ${ }^{N 128}$ (Fig. 2D). In addition to these polar interactions observed in the structure, the $\mathrm{N}$ - and $\mathrm{C}$-terminal extensions of $\mathrm{ATRX}_{\mathrm{DBM}}$ may also contribute to binding with $\mathrm{DAX} \mathrm{X}_{\mathrm{DHB}}$ through electrostatic interactions. Calculation of the electrostatic potential of $D A X X_{D H B}$ shows that the amphipathic helix of ATRX $\mathrm{DBM}_{\mathrm{DM}}$ is clamped by two basic patches of DAXX (Fig. 2E). Correspondingly, the $\mathrm{N}$ and $\mathrm{C}$-terminal extensions of $\mathrm{ATRX}_{\mathrm{DBM}}$ are rich in acidic residues (Fig. 2E). Although the $\mathrm{N}$ - and $\mathrm{C}$-terminal extensions are absent from the current structure, the close spatial disposition of these acidic extensions of $A T R X_{D B M}$ and basic patches of $D A X X_{D H B}$ strongly suggest that the acidic regions of $A T R X_{D B M}$ are associated with $D A X X_{D H B}$ through
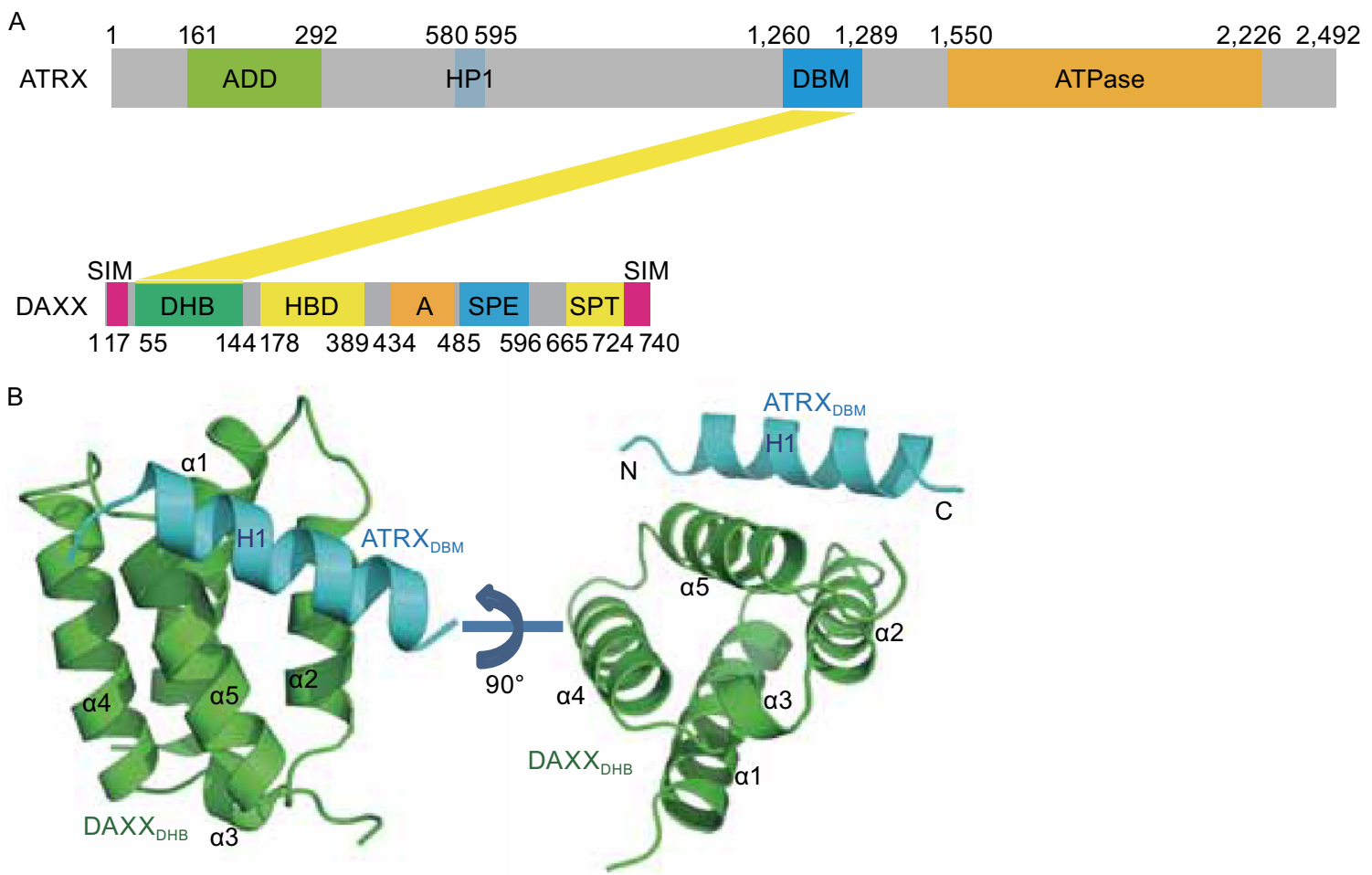

Figure 1. The structure of the $\mathrm{DAXX} \mathrm{DHB}_{\mathrm{DH}}-\mathrm{ATRX} \mathrm{DBM}_{\mathrm{DB}}$ complex. (A) Domain organization of the ATRX and DAXX. ADD, ATRXDNMT1-DNMT1L domain; HP1, HP1-binding motif; DBM, DAXX binding motif; ATPase, ATPase domain; SIM, Sumo-interaction motif; DHB, DAXX helical bundle; HBD, histone binding domain; Acidic, segment rich in Glu/Asp residues; SPE, segment rich in Ser/Pro/Glu residues; SPT, segment rich in Ser/Pro/Thr residues. (B) Two orthogonal views of the DAXX $X_{D H B}-A T R X_{D B M}$ complex. $\mathrm{DAXX} \mathrm{DHB}_{\mathrm{DHB}}$ is colored in green and ATRX $\mathrm{DBM}_{\mathrm{DM}}$ is colored in cyan. 
electrostatic interactions. To investigate the roles of these extensions, the effect of $\mathrm{N}$ - or $\mathrm{C}$-terminal truncation of ATRX $X_{\text {DBM }}$ was examined by ITC assays. Although $C$-terminal truncation had no effect on $D A X X_{D H B}-A T R X_{D B M}$ interaction, deletion of $\mathrm{N}$-terminal eight residues resulted in a $\sim 60$-fold decrease in the affinity between $\mathrm{DAX} \mathrm{X}_{\mathrm{DHB}}$ and $\mathrm{ATRX}_{\mathrm{DBM}}$ (Fig. 2F), indicating that the $\mathrm{N}$-terminal extension is essential for strong binding between $\mathrm{DAX} \mathrm{X}_{\mathrm{DHB}}$ and $\mathrm{ATR} \mathrm{X}_{\mathrm{DBM}}$. Mutations of acidic residues in the $\mathrm{N}$-terminal extension of $A T R X_{D B M}$ also weakened the interaction between $D A X X_{D H B}$ and $A T R X_{D B M}$ (Fig. 2F), further underscoring the importance of the $\mathrm{N}$-terminal-extension-mediated electrostatic interactions. Taken together, these extensive hydrophobic, electrostatic, and hydrogen-bonding interactions ensure a stable association between DAXX and ATRX.

DAXX is a scaffold protein that interacts with more than 50 proteins with diverse roles (Lindsay et al., 2008). The DAXX helical bundle (DHB) domain has been reported to interact with ATRX, RASSF1C, MDM2, HAUSP, P53, P63, and P73 (Escobar-Cabrera et al., 2010; Gostissa et al., 2004; Tang et al., 2006; Tang et al., 2004). The molecular mechanism by which DAXX $X_{D H B}$ recognizes different partners remains poorly understood. Here we compared complex structures of DAXX $X_{D H B}-A T R X_{D B M}$ and DAXX $X_{D H B}-R A S S F 1 C_{D B M}$. ATRX $X_{D B M}$ and RASSF1C $C_{D B M}$ both exist as amphipathic helices and bind to the same groove between helices $\alpha 2$ and $\alpha 5$ of DAXX $X_{D H B}$ (Fig. 2G). Although these two DBMs show low sequence homology, key residues involved in hydrophobic contacts are conserved (Fig. 2H and 2I). ATRX L1276, L1277, and I1280 occupy positions corresponding to those of L31, F35, Y34 of RASSF1C, respectively (Fig. 2H). These structural equivalent residues interact with the same panel of hydrophobic residues of DAXX (Fig. $2 \mathrm{H}$ ). Notwithstanding these similarities, there are substantial structural differences between ATRX $X_{D B M}$ and RASSF $1 C_{\text {DBM. }}$. First, they exhibit distinct orientations within the complex structures. ATRX $\mathrm{DBM}_{\mathrm{DM}}$ extends across the $\alpha 2$ and $\alpha 5$, while RASSF $1 C_{D B M}$ is anti-parallel to $\alpha 2$ and $\alpha 5$ of DAXX $X_{D H B}$ (Fig. 2G). In this way, these two DBM helices are almost perpendicular to each other. Second, both $\mathrm{N}$ - and $\mathrm{C}$-terminal extensions of $\mathrm{ATRX}_{\mathrm{DBM}}$ are acidic in nature, while RASSF $1 C_{D B M}$ has a negatively charged $\mathrm{N}$-terminal extension and a positively charged C-terminal tail. Due to the topological difference between $A T R X_{D B M}$ and RASSF1C ${ }_{D B M}$, the basic C-terminal tail of RASSF1C ${ }_{D B M}$ is close to the basic patch of $D A X X_{D H B}$, which is where the acidic $\mathrm{N}$-terminal extension of $A T R X_{D B M}$ binds (Fig. $2 E$ and $2 \mathrm{G})$. The basic $\mathrm{C}$-terminal tail of RASSF1 $\mathrm{C}_{\mathrm{DBM}}$ may interfere with the otherwise strong binding to $D A X X_{D H B}$. This may explain the relatively low binding affinity between $D A X X_{D H B}$ and RASSF1C DBM $_{\text {R }}\left(K_{\mathrm{d}}=65 \mu \mathrm{mol} / \mathrm{L}\right)$ (Escobar-Cabrera et al., 2010).

In summary, the structural characterization of the $\mathrm{DAXX}_{\mathrm{DHB}}$ domain in complex with $\mathrm{ATR}_{\mathrm{DBM}}$ provides a molecular framework for understanding the interaction between DAXX and ATRX. The DAXX-ATRX interaction is a crucial link to bridge the chaperone-activity domain of DAXX and the remodeling-activity domain of ATRX together to deposit H3.3 into heterochromatin foci. The structural model and mutagenesis data presented here also provide an opportunity to dissect the functional consequences of specific disruption of DAXX-ATRX in vivo. Although there are a few of disease mutations identified in regions of $A T R X_{D B M}$ and $D A X X_{D H B}$, none of these mutations seems to affect DAXX-ATRX interaction (Fig. S3). Why the DAXX-ATRX interface is not susceptible to disease mutations needs further investigation. Moreover, our structural analyses of $D A X X_{D H B}-A T R X_{D B M}$ and DAXX $X_{D H B}-R A S S F 1 C_{D B M}$ indicate that $\mathrm{DAX} \mathrm{X}_{\mathrm{DHB}}$ is a general protein-interaction domain with sufficient structural plasticity to accommodate DBMs from different interaction partners. Given that the topological relationships of these DBMs are completely different, at this stage, it would be difficult to detect the hidden similarities among these DBMs based solely on sequence information, without $3 \mathrm{D}$ structural information. As more $\mathrm{DAX} \mathrm{X}_{\mathrm{DHB}}$-interaction partners are identified and their structures become available, it should be possible to identify the conserved features of these interaction partners in the future.

\section{FOOTNOTES}

We thank staffs from BL18U1 and BL19U1 beamlines at NCPSS and Shanghai Synchrotron Radiation Facility (SSRF) for help with crystal data collection. We are extremely grateful to National Center for Protein Sciences Shanghai (Protein Expression and Purification system, NMR system, Mass Spectrometry) for their instrument support and technical assistance. This work was supported by grants from the Strategic Priority Research Program of the Chinese Academy of Sciences (XDB08010201) to Y.C., the Ministry of Science and Technology of China (2013CB910401 to Y.C.), the National Natural Science Foundation of China (31470737 and 31670748 to Y.C.), and the Basic Research Project of Shanghai Science and Technology Commission (14JC1407200 to Y.C.).

Coordinates and structure factors have been deposited in the Protein Data Bank under accession codes $5 Y 18$ (DAXX $\mathrm{DHB}^{-}$ ATRX 
A

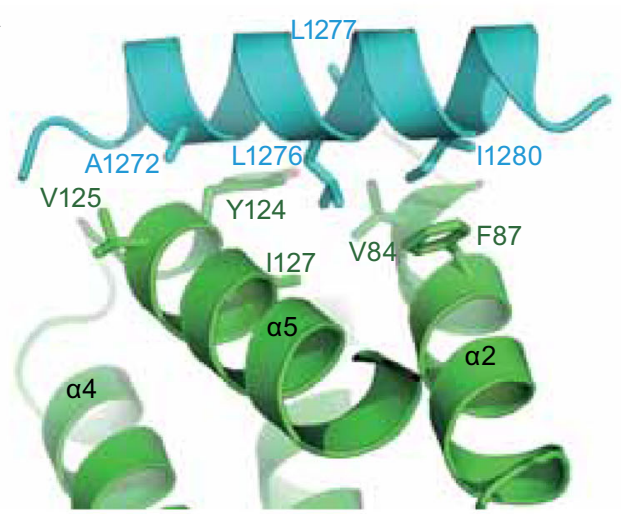

D

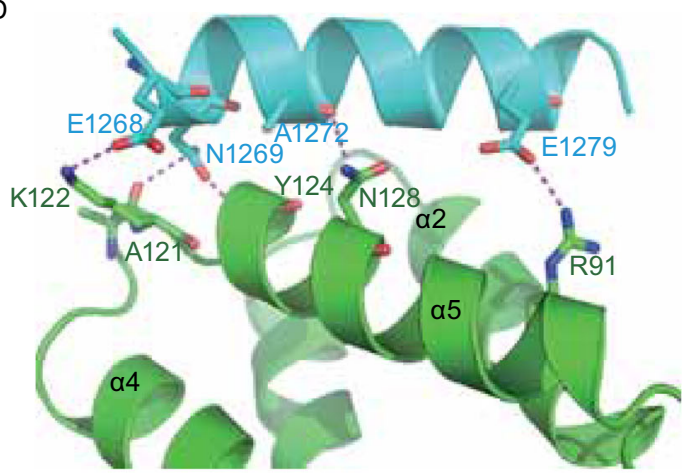

B
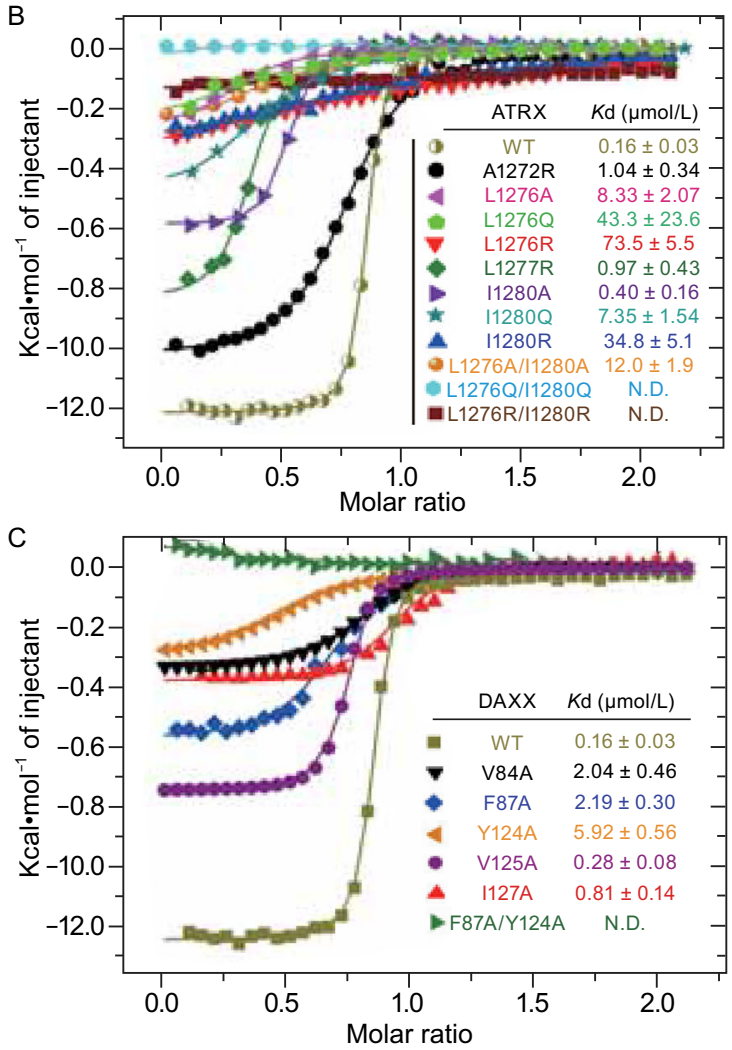

E

$\mathrm{F}$

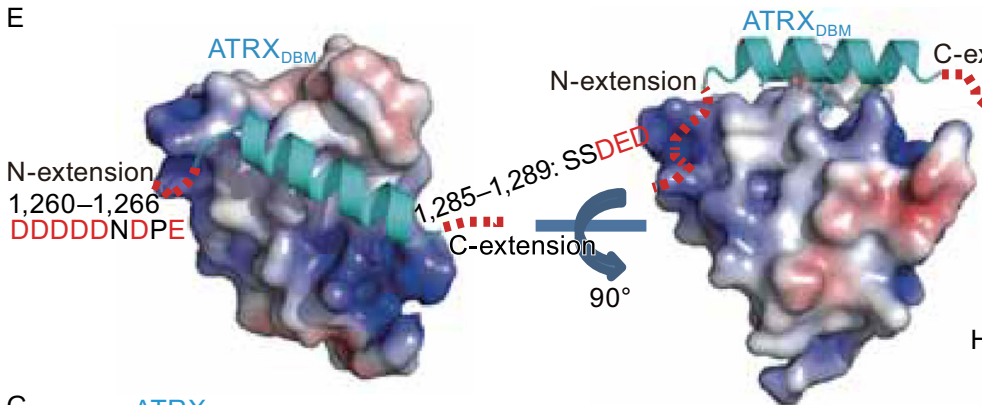

$\mathrm{H}$

\begin{tabular}{cccc} 
& $F$ & ATRX & DAXX \\
\cline { 2 - 4 } extension & $K d(\mu \mathrm{mol} / \mathrm{L})$ \\
\hline $1,260-1,289$ & WT & $0.16 \pm 0.03$ \\
& $1,268-1,289$ & WT & $10.3 \pm 0.5$ \\
$1,260-1,285$ & WT & $0.15 \pm 0.02$ \\
$1,260-1,289$ M1 & WT & $0.85 \pm 0.10$ \\
$1,260-1,289$ M2 & WT & $1.75 \pm 0.23$
\end{tabular}

G
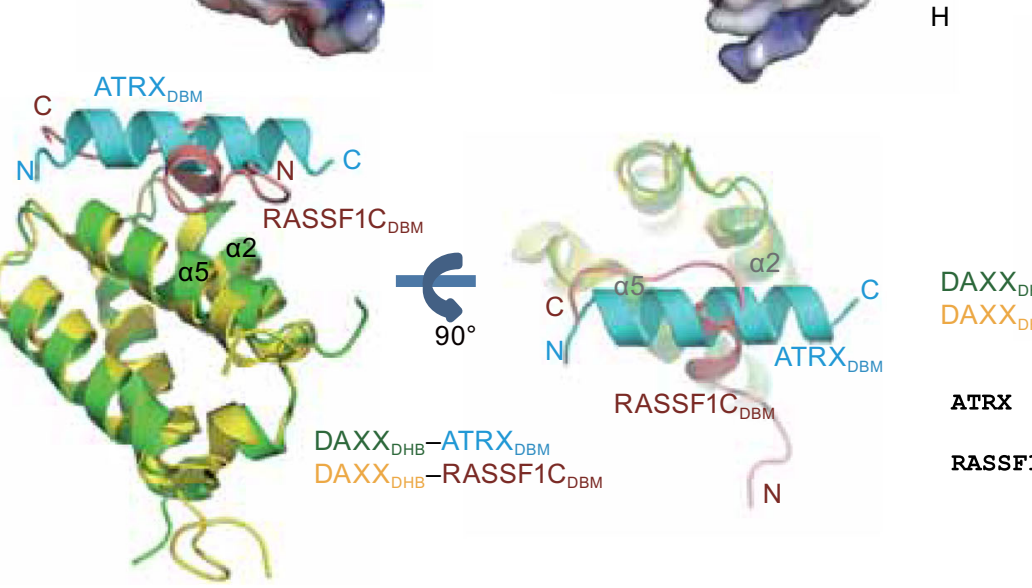

M1: D1260A/D1261A/D1262A/D1263A/D1264A M2: D1266A/E1268A

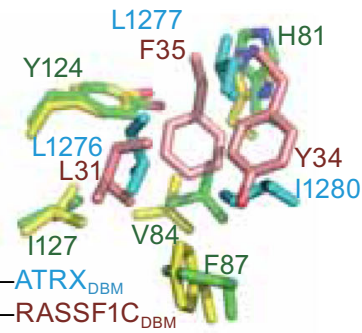

ATRX DDDDDDNDPENRIAKKMLLEETKANLSSDED

RASSF1C

SQEDSDSELEQYFTARTSLARRPRR 
4 Figure 2. The interfaces between DAXX $X_{D H B}$ and $A T R X_{D B M}$. (A) Details of hydrophobic contacts in the DAXX $X_{D H B}-A T R X_{D B M}$ interface. The contacting residues are presented as ball-andstick models. DAXX residues are colored in green and ATRX residues are colored in cyan. ( $B$ and $C$ ) Effects of mutations in the $\mathrm{ATRX}_{1,253-1,326}(\mathrm{~B})$ and $\mathrm{DAXX}_{55-144}(\mathrm{C})$ on the interaction between DAXX and ATRX analyzed by ITC assays. The $K_{d}$ values for WT and mutants were indicated. "N.D." stands for "Not Detectable". (D) Details of salt bridge and hydrogenbonding interactions between DAXX $X_{\mathrm{DHB}}$ and ATRX $\mathrm{DBM}_{\mathrm{DM}}$, shown as dashed red lines. (E) The $\mathrm{N}$ - and C-terminal extensions of $A T R X_{D B M}$ may interact with $D A X X_{D H B}$. DAXX $X_{D H B}$ is shown in surface representation and colored according to its electrostatic potential (positive potential, blue; negative potential, red). The absent $\mathrm{N}$ - and $\mathrm{C}$-terminal extensions cannot be modeled unambiguously. The red dashed lines indicate possible location of these missing extensions for illustration purpose. (F) Effects of truncations and mutations of ATRX $X_{D B M}$ on DAXX-ATRX interactions shown by ITC assays. (G) Superimposition of $\mathrm{DAXX}_{\mathrm{DHB}}-\mathrm{ATRX}_{\mathrm{DBM}}$ and DAXX $\mathrm{DHB}_{\mathrm{DH}}-\mathrm{RASSF}_{\mathrm{C}} \mathrm{C}_{\mathrm{DBM}}$ (PDB: $2 K Z U$ ) structures shown in two orthogonal views. $D A X X_{D H B}$ in $\mathrm{DAXX}_{\mathrm{DHB}}-\mathrm{ATRX}_{\mathrm{DBM}}$ complex, green; $\mathrm{ATRX}_{\mathrm{DBM}}$, cyan; $\mathrm{DAXX}_{\mathrm{DHB}}$ in DAXX $\mathrm{DHB}_{\mathrm{BH}}-\mathrm{RASSF} 1 \mathrm{C}_{\mathrm{DBM}}$ complex, yellow; RASSF1 DBM $_{\text {, red. }}(H)$ The conserved hydrophobic interfaces in two structures. The structural equivalent residues are indicated. (I) The sequence alignment of ATRX $X_{D B M}$ and RASSF1C $C_{D B M}$ shows low similarity. The structural equivalent residues are not sequentially aligned.

Y. C, conceived this study. X. W., Y. Z. and J. Z. purified the proteins and performed crystallization. X. W. and Y.C. collected the data and carried out crystallography analyses. X.W. performed ITC analyses. Y.C., X.W and Y.Z wrote the manuscript.

Xiaoman Wang, Yiyue Zhao, Jian Zhang, and Yong Chen declare that they have no conflict of interest.

\section{Xiaoman Wang ${ }^{1}$, Yiyue Zhao ${ }^{1,2}$, Jian Zhang ${ }^{1}$, Yong Chen ${ }^{1,2 \bowtie}$ \\ 1 State Key Laboratory of Molecular Biology, National Center for Protein Science Shanghai, Shanghai Science Research Center, CAS Center for Excellence in Molecular Cell Science, Shanghai Institute of Biochemistry and Cell Biology, Chinese Academy of Sciences, University of Chinese Academy of Sciences, Shanghai 201210, China \\ 2 School of Life Science and Technology, Shanghai Tech University, Shanghai 201210, China \\ $\bowtie$ Correspondence: yongchen@sibcb.ac.cn (Y. Chen)}

\section{OPEN ACCESS}

This article is distributed under the terms of the Creative Commons Attribution 4.0 International License (http://creativecommons.org/

Electronic supplementary material The online version of this article (doi:10.1007/s13238-017-0462-y) contains supplementary material, which is available to authorized users. licenses/by/4.0/), which permits unrestricted use, distribution, and reproduction in any medium, provided you give appropriate credit to the original author(s) and the source, provide a link to the Creative Commons license, and indicate if changes were made.

\section{REFERENCE}

Clynes D, Higgs DR, Gibbons RJ (2013) The chromatin remodeller ATRX: a repeat offender in human disease. Trends Biochem Sci 38:461-466

Dyer MA, Qadeer, Z.A., Valle-Garcia, D., and Bernstein E (2017) ATRX and DAXX: Mechanisms and Mutations. Cold Spring Harb Perspect Med 7 .

Elsasser SJ, Huang $\mathrm{H}$, Lewis PW, Chin JW, Allis CD, Patel DJ (2012) DAXX envelops a histone H3.3-H4 dimer for H3.3-specific recognition. Nature 491:560-565

Escobar-Cabrera E, Lau DK, Giovinazzi S, Ishov AM, McIntosh LP (2010) Structural characterization of the DAXX N-terminal helical bundle domain and its complex with Rassf1C. Structure 18:16421653

Goldberg AD, Banaszynski LA, Noh KM, Lewis PW, Elsaesser SJ, Stadler S, Dewell S, Law M, Guo X, Li X et al (2010) Distinct factors control histone variant $\mathrm{H} 3.3$ localization at specific genomic regions. Cell 140:678-691

Gostissa M, Morelli M, Mantovani F, Guida E, Piazza S, Collavin L, Brancolini C, Schneider C, Del Sal G (2004) The transcriptional repressor hDaxx potentiates p53-dependent apoptosis. J Biol Chem 279:48013-48023

Iwase S, Xiang B, Ghosh S, Ren T, Lewis PW, Cochrane JC, Allis CD, Picketts DJ, Patel DJ, Li H et al (2011) ATRX ADD domain links an atypical histone methylation recognition mechanism to human mental-retardation syndrome. Nat Struct Mol Biol 18:769-776

Law MJ, Lower KM, Voon HP, Hughes JR, Garrick D, Viprakasit V, Mitson M, De Gobbi M, Marra M, Morris A et al (2010) ATR-X syndrome protein targets tandem repeats and influences allelespecific expression in a size-dependent manner. Cell 143:367-378

Lewis PW, Elsaesser SJ, Noh KM, Stadler SC, Allis CD (2010) Daxx is an H3.3-specific histone chaperone and cooperates with ATRX in replication-independent chromatin assembly at telomeres. Proc Natl Acad Sci USA 107:14075-14080

Lindsay CR, Morozov VM, Ishov AM (2008) PML NBs (ND10) and Daxx: from nuclear structure to protein function. Front Biosci 13:7132-7142

Liu CP, Xiong C, Wang M, Yu Z, Yang N, Chen P, Zhang Z, Li G, Xu RM (2012) Structure of the variant histone H3.3-H4 heterodimer in complex with its chaperone DAXX. Nat Struct Mol Biol 19:1287-1292

Tang J, Wu S, Liu H, Stratt R, Barak OG, Shiekhattar R, Picketts DJ, Yang X (2004) A novel transcription regulatory complex containing death domain-associated protein and the ATR-X syndrome protein. J Biol Chem 279:20369-20377

Tang J, Qu LK, Zhang J, Wang W, Michaelson JS, Degenhardt YY, El-Deiry WS, Yang $X(2006)$ Critical role for Daxx in regulating Mdm2. Nat Cell Biol 8:855-862

Watson LA, Goldberg H, Berube NG (2015) Emerging roles of ATRX in cancer. Epigenomics 7:1365-1378 